\title{
Relevante Zeitbereiche intralogistischer Datenströme - Grundlagen für die Optimierung im laufenden Betrieb
}

\author{
Dipl.-Ing. David Wustmann, Dipl.-Wirtsch.-Ing. Robert Schmaler, \\ Prof. Dr.-Ing. habil. Thorsten Schmidt \\ Technische Universität Dresden \\ Professur für Technische Logistik
}

\begin{abstract}
Materialflussrechner in komplexen Materialflusssystemen generieren eine große Anzahl an Daten zu systeminternen Ereignissen. Deren Informationspotential wird derzeit aufgrund des enormen Datenumfangs nur begrenzt genutzt. Für online durchgeführte Analysen ist sogar von einer stetig wachsenden Datenmenge auszugehen. Dieser Beitrag beschreibt einen Ansatz, diese Datenmenge in Zeitbereiche einzuteilen und die valide Echtzeit-Berechnung statistischer Prozesskenngrößen zu gewährleisten. Durch die Beachtung signifikanter Zustandsänderungen im System bezüglich der Echtzeit-Analyse können statistische Abweichungen in systeminternen Prozessen erkannt werden. Damit wird die Grundlage für eine Optimierung im laufenden Betrieb gelegt, um ggf. lokalen Prozessabweichungen entgegenwirken zu können, bevor diese Auswirkungen auf das Gesamtsystem haben.
\end{abstract}

\section{$1 \quad$ Einleitung}

Die genaue Kenntnis über temporäre und lokale Prozessabweichungen ist eine wesentliche Voraussetzung für jede Systemoptimierung. Prozessabweichungen liegen vor, wenn die betrachteten (Teil-) Prozesse vom Normal-, Soll- bzw. Optimalfall $^{1}$ abweichen. Ereignisdaten komplexer Materialflusssysteme beschreiben in ihrer Gesamtheit das Verhalten des Systems sowie seine systeminternen Prozesse. Nur selten werden diese enormen Datenmengen aber für eine Prozessanalyse ${ }^{2}$ genutzt, um systeminterne Schwachstellen oder Leistungsreserven zu identifizieren [Tol07], da dies ohne numerischer Ansätze nur sehr stark eingeschränkt möglich ist.

Für online durchgeführte Prozessanalysen ist darüber hinaus von einer stetig wachsenden Datenmenge ohne abgegrenzten Ereignishorizont auszugehen. Die das Prozessverhalten beschreibenden Kennwerte und statistischen Größen müssen stets das reale Systemverhalten widerspiegeln. Für die valide Prozessanalyse im laufenden Betrieb ist es demnach notwendig, den Datenstrom in relevante Zeitbereiche zu unterteilen. Beispielsweise sinkt die Aussagekraft statistischer

\footnotetext{
${ }^{1}$ Normalfall: statistisches Verhalten; Optimalfall: bestmögliche Prozessparameter; Sollfall: Vorgabewerte.

2 Im Folgenden wird synonym für Prozessanalyse auch Materialflussdiagnose oder numerische Ereignisdatenauswertung verwendet, da ausschließlich Transportprozesse mit Softwareunterstützung analysiert werden.
} 
Prozesskenngrößen mit steigendem Datenumfang (Abbildung 1), insbesondere bei signifikanten Zustandsänderungen (Lastzyklen etc).

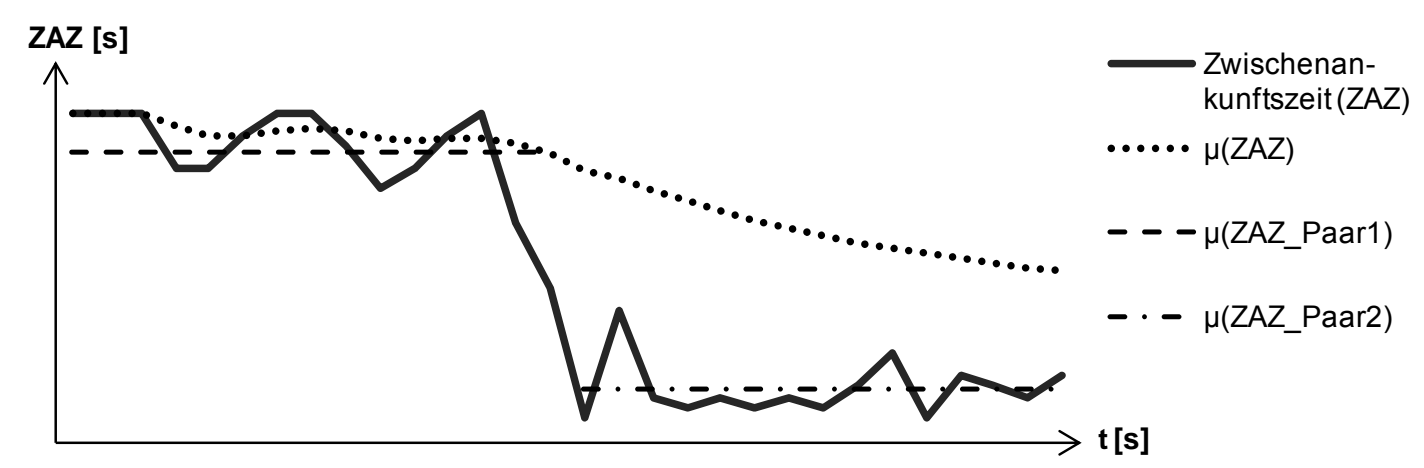

Abbildung 1: steigender Datenumfang - sinkender Informationsgehalt statistischer Werte

Der Beitrag zeigt eine Methode zur Identifizierung relevanter Zeitbereiche im Verhalten eines Systems anhand von Ereignisdaten. Der stetige Datenstrom soll demnach an geeigneter Stelle in Cluster unterteilt werden (Abbildung 2).

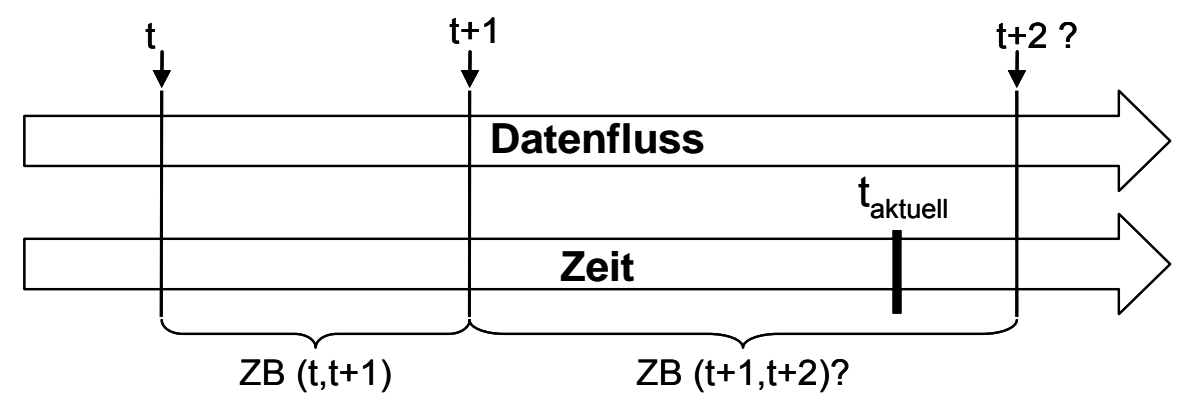

Abbildung 2: Zeitbereiche intralogistischer Datenströme - Zielsetzung

Im Folgenden werden der Stand der Technik sowie die eigenen Entwicklungen zur Materialflussdiagnose als Ausgangssituation für die in diesem Beitrag beschriebene Methode zur Definition relevanter Zeitbereiche dargestellt.

\section{Ausgangssituation}

\subsection{Numerische Ereignisdatenauswertung}

Das Generieren von Ereignisdaten aus Simulationen und deren Analyse ist nach Kemper (2007) ein mächtiges Werkzeug zur Fehlersuche in Simulationsmodellen und wird derzeit hauptsächlich zur Modellverifikation und -validierung verwendet [Kem07]. Better et al. (2007) und Kemper/Tepper (2007) schlagen beispielsweise eine Kombination von Dynamic-Data-Mining- und Optimierungs-Techniken vor, jedoch werden ausschließlich Modellfehler gesucht [Bet07; Kem07]. Die Analyse systeminterner Prozesse beschränkt sich fast ausschließlich auf die grafische Visualisierung eines reduzierten Datensatzes, um den Analysten zu unterstützen [Kem05; Lee04 \& Wöl00]. Neben diesen grafisch geprägten Ansätzen finden sich in anderen Domänen auch numerische Methoden, beispielsweise zur ereignisbasierten Analyse von Geschäftsprozessen [Aal04, Roz09]. Der Fokus des „business process 
mining" liegt jedoch in erster Instanz im Erstellen adäquater Modelle und dem Erkennen von relevanten Prozessschritten [Gün06; Las08].

Eigene Untersuchungen zeigen die Machbarkeit und das Potential der numerischen Ereignisdatenauswertung für komplexe Materialflusssysteme [Vas10; Sch10]. Die entwickelten Kenngrößen, Algorithmen sowie verwendeten statistischen Verfahren wurden in einem Prototyp implementiert und getestet. Dieser ermöglicht die schnelle Offline-Analyse und -Bewertung systeminterner Prozesse in spurgebundenen Transportsystemen basierend auf den drei Standard-Ereignisinformationen [Wus09]:

- Ort des Ereignisses

- ID des Fördergutes

- Zeitstempel des Ereignisses für eine abgrenzte Ereignisdatenmenge.

Aus den Ereignisattributen werden ein Knoten-Kanten-Modell des Systems generiert und primäre Prozess- sowie statistische Kennwerte berechnet. Abbildung 3 zeigt ein Knoten-Kanten-Modell aus 0,5 Mio. Ereignissen einer realen Anlage mit Wärmebildvisualisierung für identifizierte Prozessabweichungen an den Ereignisorten im betrachteten Zeithorizont (hier Graustufen: dunkel für kritisch bis hell für unkritisch).

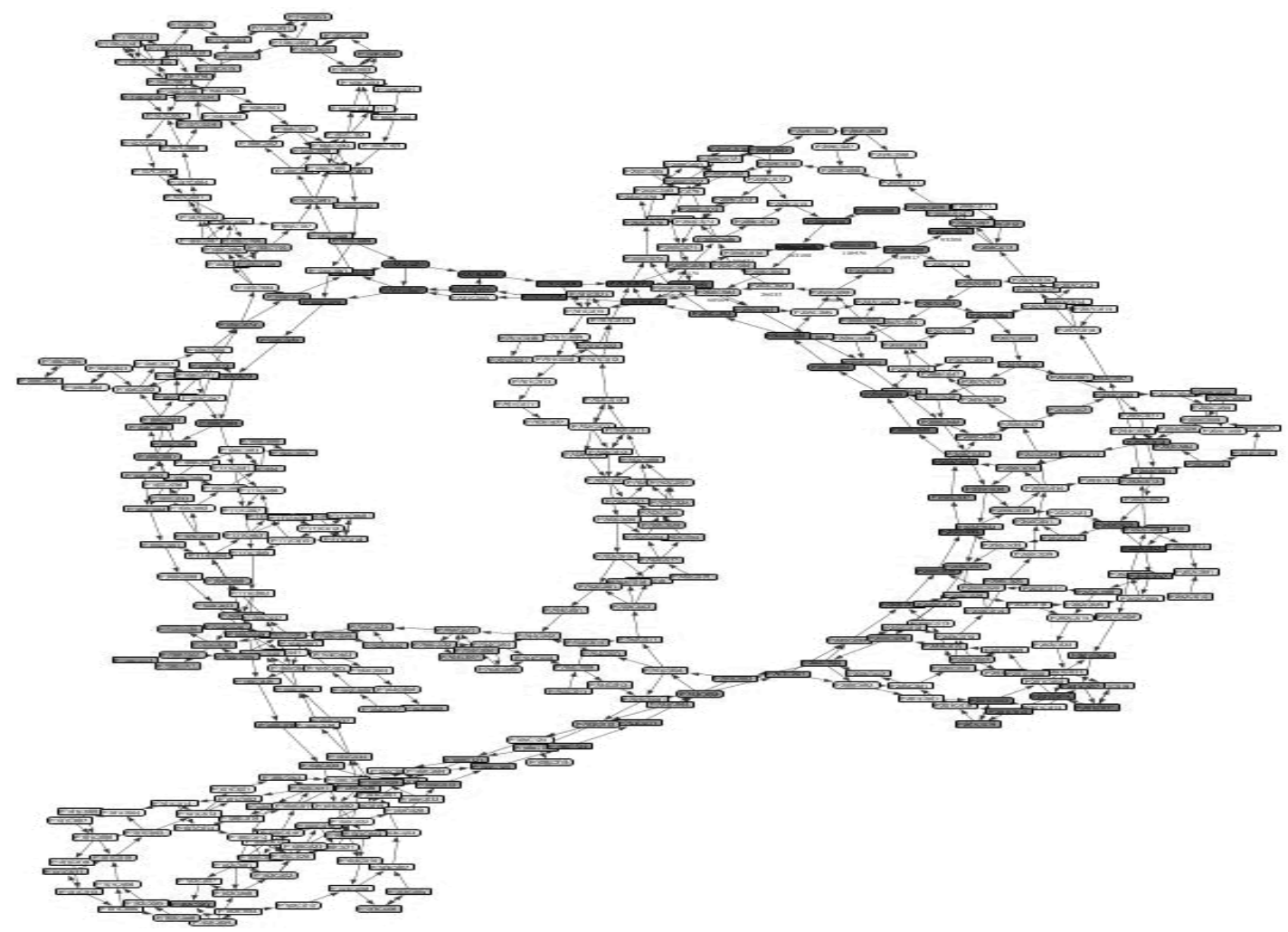

Abbildung 3: Knoten-Kanten-Modell; Visualisierung identifizierter interner Prozessabweichungen

Auf Basis der drei Ereignisinformationen werden unter anderem die für diesen Beitrag zur Bildung variabler Zeitbereiche relevanten (Primär-) Kenngrößen

- Nutzungsdauer (ND) 
- ZeitimSystem ( ZiS ) (Zeit eines Fördergutes bis zum Ereignisort)

- Zwischenankunftszeit (ZAZ)

- Kantenlast $(K L)$ (Fördergutanzahl zum Zeitpunkt $t$ auf einer Kante) und der

- Fördergutzähler ( FgZ)

sowie die jeweiligen statistischen Kenngrößen wie beispielsweise

- Variationskoeffizienten, Standardabweichungen

- Mittelwerte (Median, Modus, arithm. Mittel)

- Minima und Maxima berechnet [vgl. Wus10].

\subsection{Online-Materialflussdiagnose}

Bereits Schenk et al. (2007) stellen fest, dass experimentelle Untersuchungen sowie ein präzises theoretisches Konzept von großer Bedeutung für die Umsetzung von Echtzeitsystemen sind [Sche07]. Hierzu ist nach Tolujew et al. (2007) die Unterscheidung verschiedener Klassen logistischer Echtzeitsysteme sinnvoll [Tol07]. Demnach bilden das Monitoring als Bestandteil des Logistikcontrollings [Wie04], das Event Management zur automatischen Erkennung vordefinierter Situationen [Ste03] sowie Frühwarnsysteme, auf Basis betriebsbegleitender Simulationen [Han05], die wesentlichen funktionellen Klassen für Echtzeitsysteme in der Logistik. Nach dem derzeitigen Stand und dem Entwicklungsziel bis hin zur Trendanalyse, ist die OnlineMaterialflussdiagnose im Logistikcontrolling und Event Management einzuordnen.

Damit die numerische Ereignisdatenanalyse online, d.h. im laufenden Betrieb, möglich ist, sind zwei Kernpunkte zu klären. Zum einen wann Daten für die laufende Analyse nicht mehr relevant sind und zum anderen wie die Validität von Prozesskenngrößen, insb. statistischer Größen zum Systemverhalten, zu gewährleisten ist. Ziel ist es, die Berechnungen zur Offline-Analyse in den Online-Fall zu überführen. Die Abbildung 4 zeigt dazu das Gesamtkonzept der OnlineMaterialflussdiagnose.

Wichtige Voraussetzung für die Online-Anwendung der Kenngrößen und Algorithmen ist die Datenclusterung. Die dynamische Definition relevanter Zeitbereiche anhand von signifikanten Systemzuständen ist dabei ein wesentlicher Bestandteil. Zusätzlich gehört, neben dem ebenso im Offline-Fall berücksichtigten statistischen Einfluss [vgl. Her92], eine Vorprüfung auf Prozessabweichungen zu diesem Methodenbaustein, denn längerfristige Abweichungen sind auch als solche zu erkennen und nicht zu einem neuen Datenabschnitt zu deklarieren.

Zunächst werden daher im folgenden Abschnitt mögliche Systemzustände und deren Auswirkungen auf prozessbeschreibende Kenngrößen erörtert. 


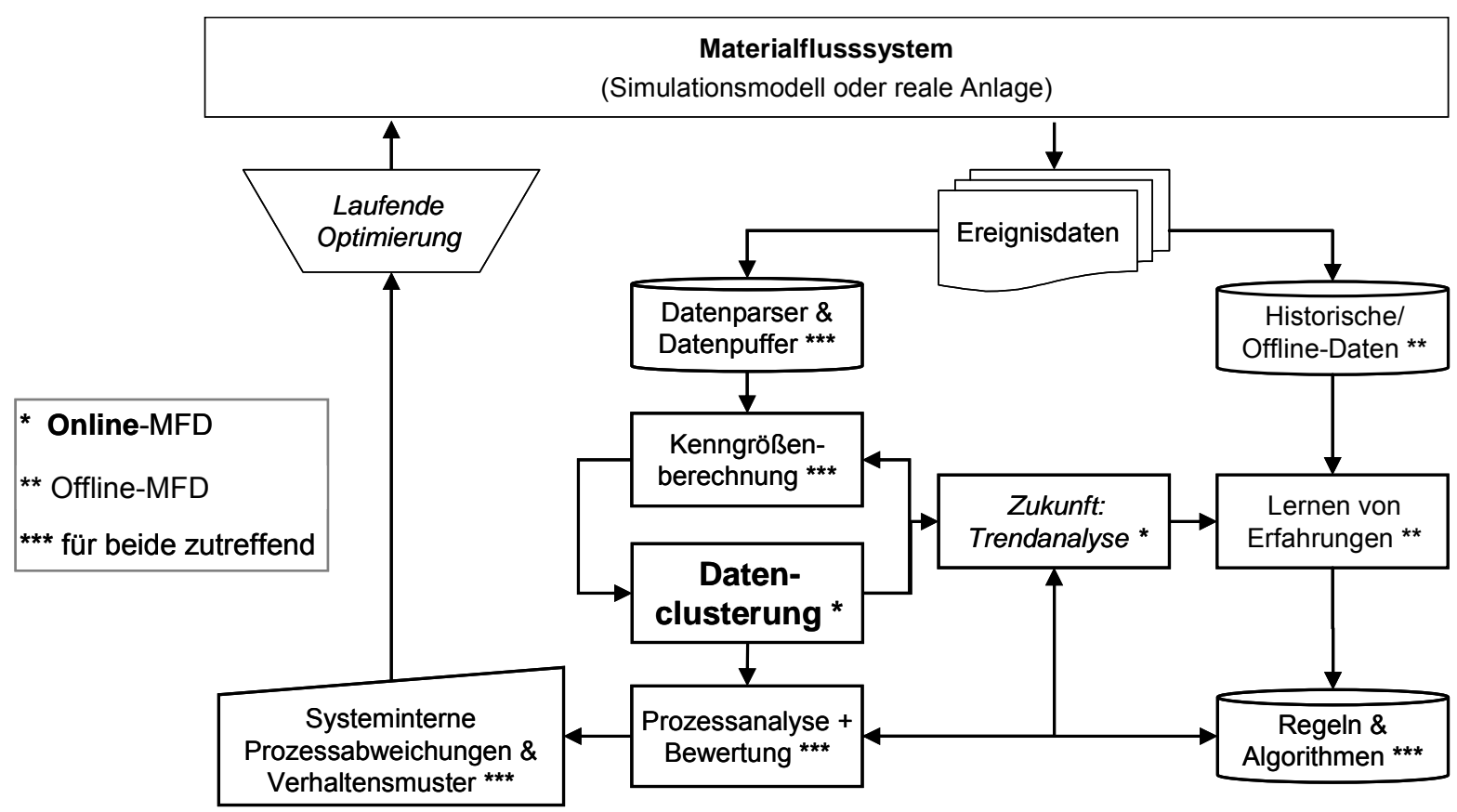

Abbildung 4: Online-Materiaflussdiagnose - Ablaufkonzept

\section{Systemzustände vs. Prozesskennwerte}

Jede signifikante Änderung im Systemzustand führt zu einer Änderung mindestens einer prozessbeschreibenden Primärkenngröße (Ausnahme: statistischer Einfluss). Im Umkehrschluss spiegeln daher die Prozesskenngrößen, speziell deren Verlauf, Zustandsänderungen wider. Für die Datenclusterung sind Zustandsänderungen, welche keiner internen Ursache unterliegen, ein geeigneter Anhaltspunkt. Tabelle 1 zeigt mögliche Zustände sowie deren Einfluss auf Kenngrößenverläufe. Je nach Art der Zustandsänderung wirkt sich diese auf die Primärkenngrößen aus.

\begin{tabular}{lllll}
\hline Systemzustände & ND & ZiS & ZAZ & KL \\
\hline Leerlauf (Fördergutstrom reißt ab) & $\rightarrow$ & $\rightarrow$ & $\uparrow$ & $\downarrow$ \\
Stillstand (keine neuen Ereignisse) & $\uparrow$ & $\uparrow$ & $\uparrow$ & $\rightarrow$ \\
Lastwechsel & $\rightarrow$ & $\rightarrow$ & $\uparrow \downarrow$ & $\uparrow \downarrow$ \\
Fördergutwechsel & $\rightarrow$ & $\rightarrow$ & $\uparrow \downarrow$ & $\uparrow \downarrow$ \\
Geschwindigkeitswechsel & $\uparrow \downarrow$ & $\uparrow \downarrow$ & $\rightarrow I \uparrow \downarrow$ & $\rightarrow$ \\
\hline
\end{tabular}

Tabelle 1: Einfluss signifikanter Systemzustände auf Kenngrößenverläufe

Mit wechselnden Lastphasen (zyklische Änderung des Transportaufkommens) ändern sich bspw. die Kenngrößen bzw. deren statistische Werte zur Zwischenankunftszeit ( $Z A Z$ ) sowie zur Kantenlast $(K L)$. Der Fördergutwechsel ist an dieser Stelle als Spezialfall des Lastwechsels zu betrachten. Ändert sich die Ausdehnung der Fördergüter in Förderrichtung signifikant, sind die Auswirkungen auf die Kenngrößen analog dem Lastwechsel. Der Wechsel zwischen Systemzuständen verläuft stets kontinuierlich, wodurch jedem neuen Zustand eine Übergangsphase vorangeht. 
Die Identifizierung der Zeitbereiche auf Basis des Leerlaufes und Stillstandes ist durch einfache Regeln möglich (Bsp. Leerlauf: $K L \rightarrow 0$ für $t \rightarrow n$ mit $n>\max (N D)$ ). Für die Datenclusterung anhand des Last- bzw. Geschwindigkeitswechsels gilt dies jedoch nicht. Zusätzlich ist sicherzustellen, dass tatsächlich eine Zustandsänderung und keine temporäre Prozessabweichung, bspw. in Form eines Dauerstaus, vorliegt.

Die Abbildung 5 zeigt detailliert den gesamten Methodenbaustein zur Datenclusterung bzw. der Zeitbereichsbildung. Treten keine Zustandsänderungen auf, kann ein Zeitbereich enormer Größe entstehen. Prozessabweichungen sind dann durch prozessbegleitende statistische Kenngrößen schlecht oder nicht zu identifizieren. Daher wird mittels des Prinzips des statischen Einfluss [vgl. Her92] nach einer spezifischen Anzahl von Ereignissen ein neuer Zeitbereich gebildet.

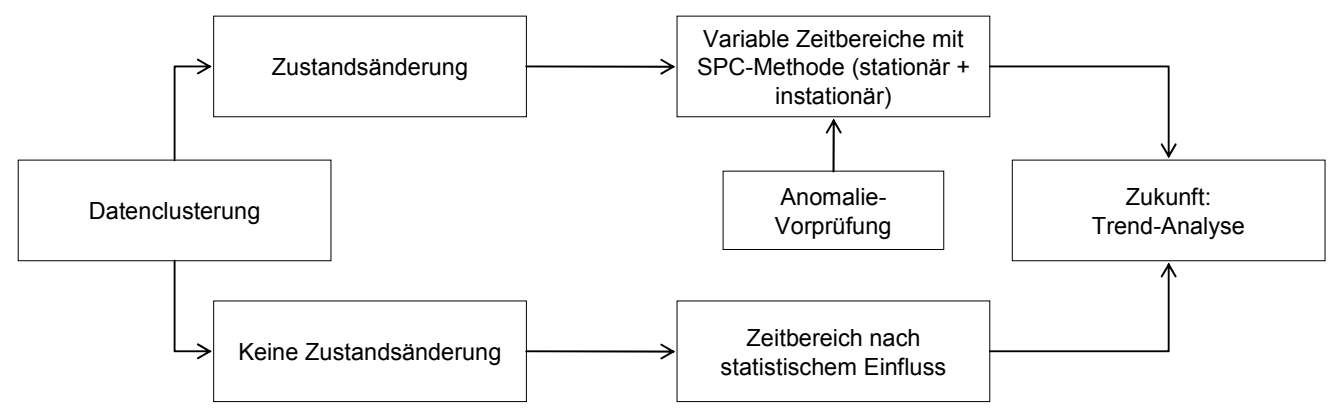

Abbildung 5: Übersicht Zeitbereichsbildung

Im folgenden Abschnitt dieses Beitrages wird die Methode zur Zeitbereichsdefinition näher beschrieben. Ausgehend von der Suche nach einem ähnlichen Verfahren wurde der geeignetste Ansatz gewählt und entsprechend adaptiert.

\section{$4 \quad$ Methode zur Definition relevanter Zeitbereiche}

\subsection{Auswahl eines geeigneten Verfahrens}

Da allgemein für die Echtzeitanalyse von Ereignisdaten keine Ansätze bekannt sind, werden die Betrachtungen auf einen verwandten Sachverhalt in der Simulation gerichtet. Üblicherweise ist in einer Simulationsstudie die Zustandsverteilung während der stationären Phase von Interesse. „Ein stochastischer Prozess $X_{t}$ wird als stationär bezeichnet, wenn die Wahrscheinlichkeit von $X_{t}$ nicht von $t$ abhängig ist." [Pag91] Befindet sich das System in einem instationären Zustand (Bsp. Anlaufphase), tritt ein systematischer Initialisierungsfehler auf. Erst die Eliminierung dieses Fehlers aus den Berechnungen garantiert korrekte Schätzer für das Modellverhalten.

Ähnlich verhält es sich bei der Übergangsphase in Folge von Zustandsänderungen. Statistische Prozesskenngrößen sollten lediglich für den Zustand repräsentative Einzelwerte enthalten. Daher wurden Ansätze aus der Simulationstheorie zur Bestimmung des Endpunktes der Warmlaufphase untersucht (Tabelle 2). 


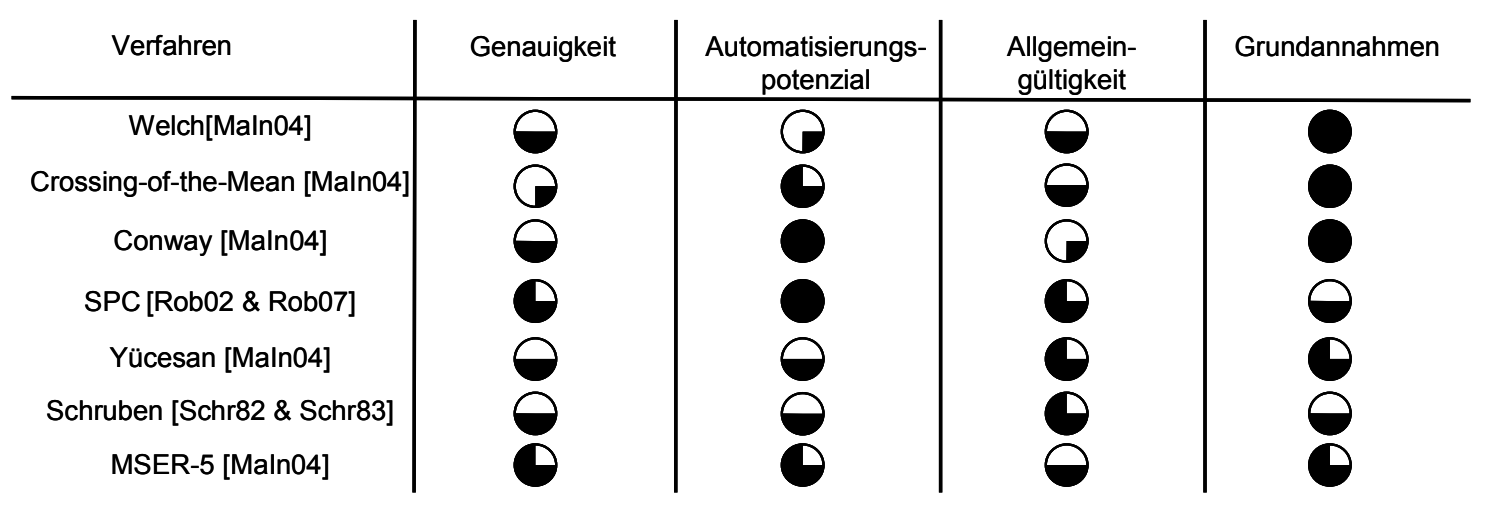

Tabelle 2: Auswertung von möglichen Verfahren zur Zeitbereichsdefinition

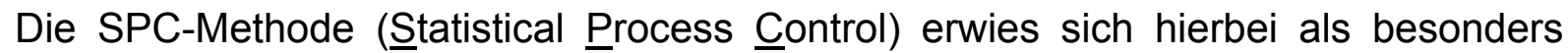
geeignet, um sowohl Übergangsphasen in Folge von Zustandsänderungen als auch die Zustandsänderung selbst anhand von Ereignisdaten zu identifizieren. Die ursprünglich aus der Qualitätssicherung stammende Statistische Prozesskontrolle zeigt, ob ein Prozess unter Kontrolle verläuft oder eine Außerkontrollsituation vorliegt. Die Gleichstellung von Außerkontrollsituationen mit Zustandsänderungen ermöglicht eine adäquate Verwendung der SPC-Methode.

\subsection{SPC-Methode für die Zeitbereichs-Definition}

Die Anwendung der SPC-Methode auf das hier behandelte Teilproblem der Materialflussdiagnose, die Datenclusterung, erfolgt in 2 Schritten: Die Ermittlung der Zustandsänderung selbst (Beginn der instationären Phase) sowie das Bestimmen der Länge der Übergangsphase (Ende der instationären Phase). Die SPC-Methode wird stets anhand jeder Primärkenngröße durchgeführt. Dafür ist die Reduzierung der Primärkenngrößen auf die Nutzungsdauer $(N D)$, Kantenlast $(K L)$ und Zwischenankunftszeit (ZAZ) in der Regel ausreichend, da die ZeitimSystem (ZiS) mit $N D$ sowie der Fördergutzähler $(F g Z)$ mit ZAZ korrelieren.

Generell stellt die SPC-Methode an die Daten zwei wichtige Anforderungen zur Durchführbarkeit: (1) unkorreliert und (2) normalverteilt. In Materialflusssystemen liegen jedoch in der Regel korrelierte Daten vor, folglich sind diese in quasiunabhängige Daten zu überführen. Ein dafür anerkanntes Verfahren ist das Batchmittelwertverfahren [Ban09]. Die Daten werden in Blöcke fester Länge, sog. Batches $(h \in H)$, aufgeteilt. Ziel ist die Generierung einer Reihe von Batchmittelwerten $\left(\mu_{h}\right)$, die Korrelation nimmt mit steigender Batchgröße tendenziell ab [Kle74]. Startwert der Batchgröße $(k)$ ist $(k=1)$.Liegen mindestens 20 Batches $(|H| \geq 20)$ vor, werden die Mittelwerte $\left(\mu_{h}\right)$ berechnet. Die 2. Hälfte der Reihe von $\mu_{h}$ wird auf Korrelation und Normalverteilung geprüft, da diese repräsentativere Schätzer für die stationäre Phase liefert. Die Anforderung (1) gilt als erfüllt, wenn die lag-1 Autokorrelation der Reihe der Batchmittelwerte $\left(\mu_{h}\right)$ einen Wert kleiner als 0,1 
annimmt. ${ }^{3}$ Wenn der $X^{2}$-Anpassungstest auf Normalverteilung ein positives Ergebnis hat, gilt auch die Anforderung (2) als erfüllt. Bei Nichteinhaltung einer der Anforderungen, muss die Batchgröße $(k)$ erhöht $(k+1)$ werden und der Zyklus wird erneut durchlaufen. Ergebnis ist eine Reihe von Batchmittelwerten $(\bar{y})$ der Anzahl $(b)$ aus Batches $(h)$ der Größe $(k)$, gebildet aus dem Ereignisvektor $(y)$ mit $m$ Einzelereignissen $(i)$ :

$$
\left.y=\left(Y_{1} ; Y_{2} ; \ldots ; Y_{m}\right) \rightarrow \bar{Y}_{h}=\frac{\sum_{i=(h-1) k+1}^{h k} Y_{i}}{k} \text { mit } h=1 ; 2 ; \ldots ; \mid \frac{m}{k}\right\rfloor \rightarrow \bar{y}=\left(\overline{Y_{1}} ; \overline{Y_{2}} ; \ldots ; \overline{Y_{b}}\right)
$$

Anschließend werden aus der 2. Hälfte von $\bar{y}$ der (Gesamt-)Mittelwert $\mu$ und die Standardabweichung $\sigma$ berechnet, um notwendigen Schätzer $\hat{\mu}$ und $\hat{\sigma}$ der sigmaGrenzen $(z)$ zu bestimmen. Daraus ergeben sich die Kontrollgrenzen $(K G)$ zur Identifikation einer Außerkontrollsituation (Zustandsänderung), wie folgt:

$$
K G=\hat{\mu} \pm \frac{z \hat{\sigma}}{\sqrt{\frac{b}{2}}} \text { für } z=1 ; 2 ; 3
$$

Nach adaptierten Regelsätzen von Montgomery \& Runger (2007) können daraufhin Zeitbereiche anhand von Zustandsänderungen definiert werden [Mon07]. Liegen zum Beispiel 4 von 6 aufeinanderfolgenden Punkten außerhalb einer $(z=2)$ sigmaKontrollgrenze wird die Anzahl der Einzelereignisse ( $i$ ) erhöht und weitere Batchmittelwerte $\left(\mu_{h}\right)$ berechnet. Sind die Daten ab einem Batch $(h)$ schließlich unter Kontrolle, ist der Endpunkt Übergangsphase bzw. der Beginn der anschließenden stationären Phase gefunden. Abbildung 6 zeigt zusammenfassend den Ablauf der SPC-Methode zur Identifizierung des Endes der Übergangsphase.

Parallel zur stationären Phase ist die SPC-Methode durchzuführen, um neue Zustandsänderungen zu erkennen. Dafür wird zunächst ebenfalls eine geeignete Batchgröße $(k)$ gesucht, die die oben genannten Anforderungen erfüllt. Die Berechnung der Batchgröße $(k)$ wird jedoch im Gegensatz zur instationären Phase an allen Batchmittelwerten $\left(\mu_{h}\right)$ der Reihe $(\bar{y})$ durchgeführt [vgl. Rob07]. Gleiches gilt für die Berechnung des Mittelwertes $\mu$ und der Standardabweichung $\sigma$ aus $\bar{y}$. Solange der Prozess unter Kontrolle ist bzw. keiner Zustandsänderung unterliegt, werden neu auftretende Ereignisse zu weiteren Batches $(h)$ zusammengefasst und in die Berechnungen einbezogen.

\footnotetext{
${ }^{3}$ Aufgrund von Abhängigkeiten höherer Ordnung ist rein statistisch aus einer geringen lag-1 Autokorrelation nicht auf unkorrelierte Daten zu schließen. Zur Anwendung der SPC-Methode ist es jedoch ausreichend [Rob02].
} 


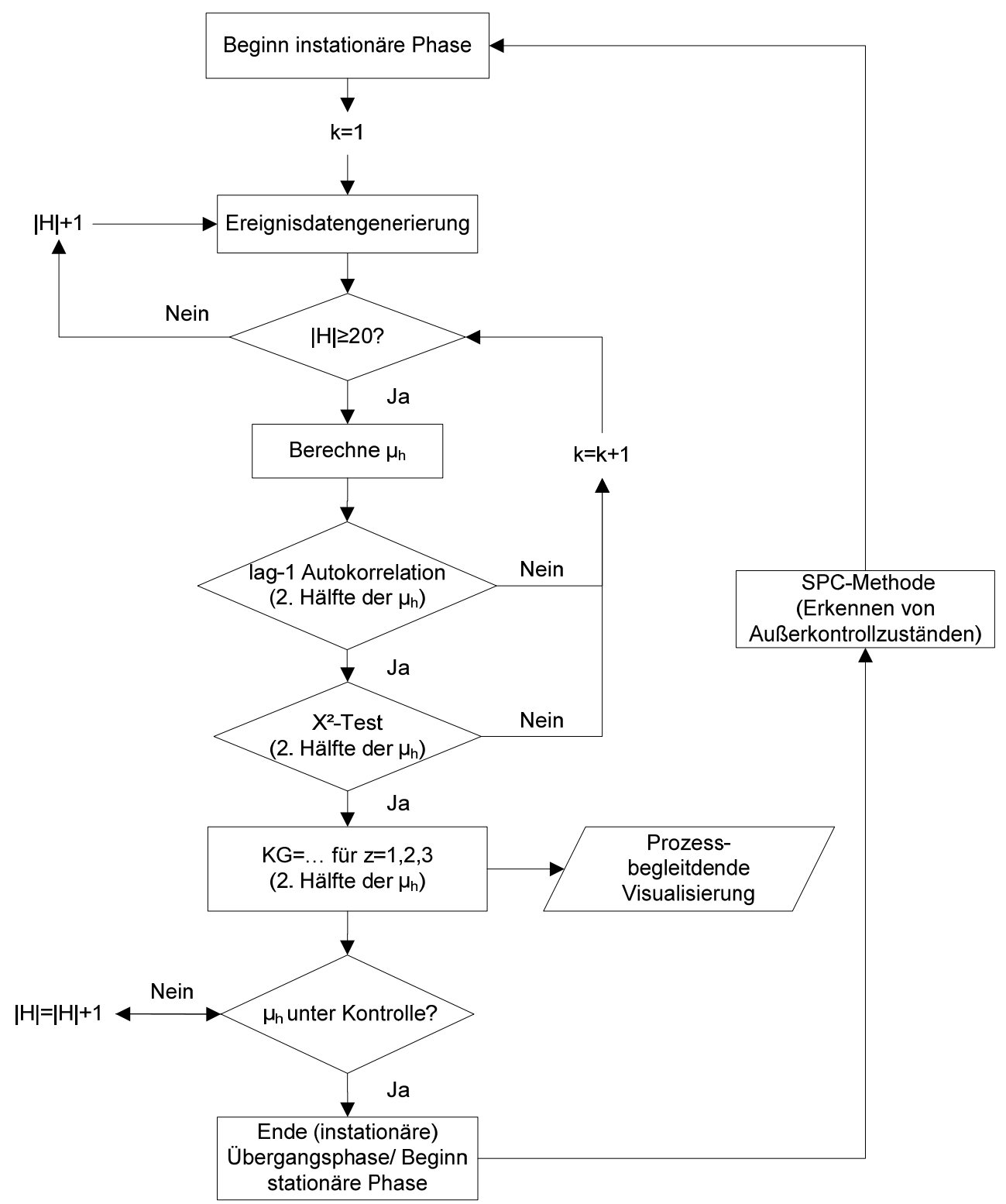

Abbildung 6: SPC-Ablaufdiagramm zur Bestimmung des instationären Datenbereichs

Gerät der Prozess bzw. ein Prozesskennwert außer Kontrolle wird erneut eine instationäre Phase und daran anschließend eine stationäre Phase ermittelt. Je nach Art der Zustandsänderung weisen die SPC-Ergebnisse der einzelnen Kenngrößen verschiedene Zeitbereiche aus.

\subsection{Validierung}

Die Validierung der SPC-Methode erfolgte zum jetzigen Zeitpunkt lediglich offline, da das dafür verwendete Software-Tool noch keine Online-Anwendungen zulässt. Dennoch konnten Datensätze aus Simulationsmodellen sowie realen Anlagen mit bis zu 200.000 Ereignissen analysiert und valide Ergebnisse erreicht werden. So konnten bspw. die in Abbildung 7 abgebildeten Produktionszyklen mit Hilfe der SPCMethode erkannt und in einzelne Zeitbereiche eingeteilt werden. 


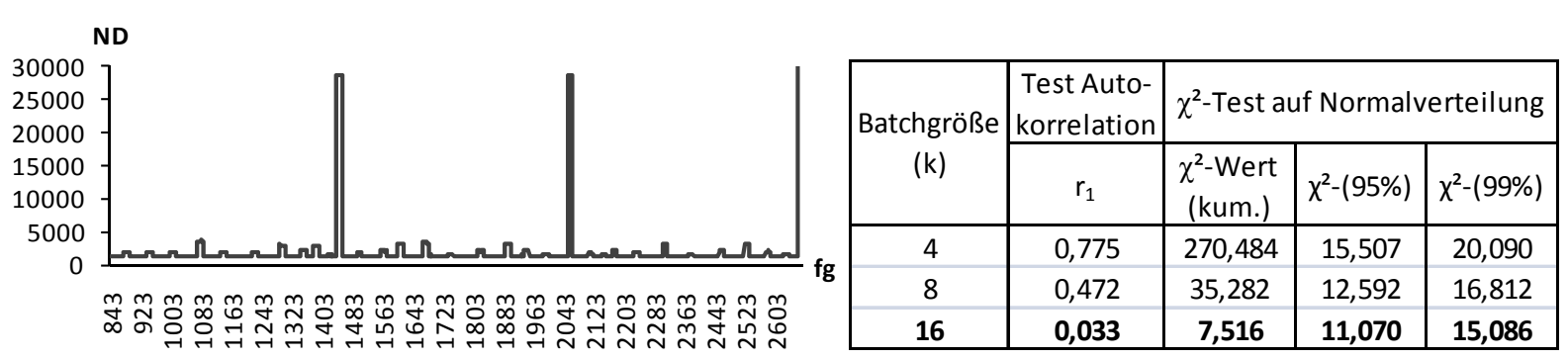

Abbildung 7: Verlauf der Nutzungsdauer (ND) einer Kante bei k=16

Die Abbildung 8 zeigt den Verlauf einer ausgewählten Übergangsphase in Folge einer Zustandsänderung (hier Systemanlauf nach einem Stopp). Die Reihe der Batchmittelwerte $\left(\mu_{h}\right)$ pendelt sich nach ungefähr einem Drittel ein und Anhäufungen von Extremwerten $\left(\mu_{h}\right.$ außerhalb einer $(z=3)$ sigma-Grenze) verschwinden. Die Ergebnisse der adaptierten Regelsätze nach [Mon07] bestätigten den grafischen Eindruck sowie auch die tatsächlichen Abläufe im System zu den Testdaten. Das Ende der Übergangsphase für das Testszenario wurde nach ca. einem Drittel der Reihe definiert. Die sich anschließenden Batchmittelwerte sind demnach bereits der nachfolgenden stationären Phase zuzuordnen.

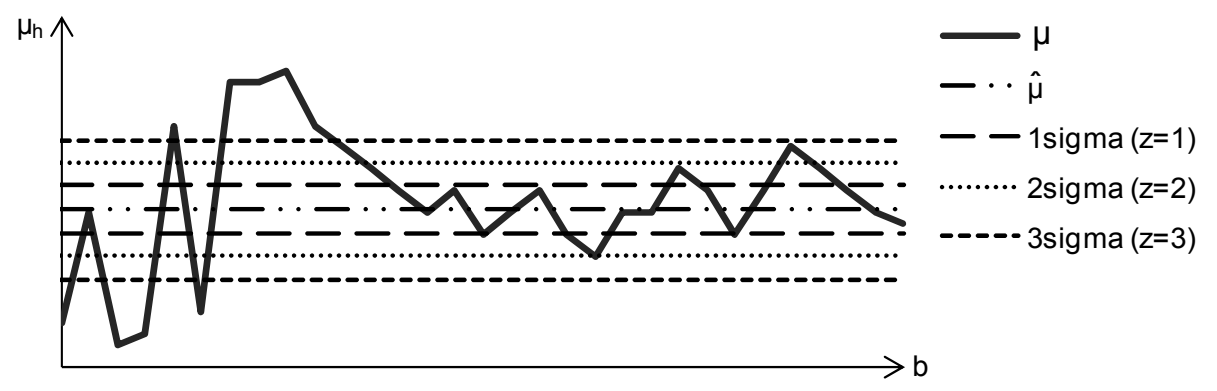

Abbildung 8: Ergebnisvisualisierung der SPC-Methode - Beispiel

\section{$5 \quad$ Zusammenfassung und Ausblick}

In diesem Beitrag wurde eine Methode zur Clusterung einer stetig wachsenden Datenmenge anhand von signifikanten Zustandsänderungen vorgeschlagen. Damit ist die Grundlage geschaffen, eine Online-Materialflussdiagnose für intralogistische Systeme anhand von Ereignisdaten durchführen zu können. Die genaue Kenntnis über temporäre und lokale Prozessabweichungen ist eine wesentliche Grundvoraussetzung für jede Systemoptimierung.

Zukünftig soll durch die Berücksichtigung von Korrelationen zwischen den Prozesskenngrößen zum einen generell der Rechenaufwand reduziert werden. Zum anderen können dadurch gegebenenfalls Zeitbereiche zusammengefasst werden. Weiteres Potential liegt darüber hinaus in der Einbeziehung zusätzlicher Ereignisinformationen für die Berechnungen. Diese sind jedoch in ihrer Verfügbarkeit sehr unterschiedlich und spielen für die gesamte Materialflussdiagnose eine entscheidende Rolle. 
Die Gesamtmethode zur numerischen Ereignisdatenauswertung wird in Zukunft zusätzlich um den Baustein der Trendanalyse erweitert. Mittels Algorithmen zur Trendmustererkennung je definierter Zeitbereiche sollen Prozessentwicklungen bzw. -abweichungen frühzeitig erkannt werden.

\section{Literatur}

[Aal04] Aalst, W. M. P.; Weijters, A. J. M.: Process mining: a research agenda. In: Computers in Industry, Bd. 53/3, S. 231-244, 2004

[Ban09] Banks, J.; Carson, J. S.; Nelson, B. L.; Nicol, D. M.: Discrete-Event System Simulation. 5. Aufl. S.450, Prentice Hall, 2009

[Bet07] Better, M.; Glover, F.; Laguna, M.: Advances in analytics: integrating dynamic data mining with simulation optimization. In: IBM Journal of Research and Development, Bd. 51/3, S. 477-487, 2007

[Gün06] Günther, C. W.; Aalst, W. M. P.: Process Mining in Case Handling Systems. In: Multikonferenz Wirtschaftsinformatik 2006 (Passau), hrsg. V. E. H. Lehner \& P. G. Kleinschmidt, S. 125-137, 2006

[Han05] Hanisch, A. ; Tolujew, J.; Schulze, T.: Initialization of online simulation models. In: WSC '05 Proceedings of the $37^{\text {th }}$ conference on Winter simulation (Orlando, Florida), S. 1795-1803, 2005

[Her92] Herz, R.; Schlichter, H.; Siegener, W.: Angewandte Statistik für Verkehrsund Regionalplaner, S. 120-121, Werner, 1992

[Kem07] Kemper, P.: A trace-based visual inspection technique to detect errors in simulation models. In: Proceedings of the 2007 Winter Simulation Conference (Washington D.C., 2007), hrsg. V. S. G. Henderson et al., S. 747-755, 2007

[Kem05] Kemper, P.; Tepper, C.: Trace based analysis of process interaction models. In: Proceedings of the 37th conference on Winter simulation (Orlando, Florida, 2005), S. 427-436, 2005

[Kem07] Kemper, P.; Tepper, C.: Automated Analysis of Simulation Traces Separating Progress from Repetitive Behavior. In: Proceedings of the Fourth International Conference on Quantitative Evaluation of Systems, S. 101-110, 2007

[Kle74] Kleijnen, J. P.: Statistical techniques in simulation. Bd. 1, hrsg. V. Marcel Decker Inc., New York, S. 459, 1974

[Las08] Lassen, K. B.; Dongen, B. F.; Aalst, V. D. W. M.: Translating Message Sequence Charts to other Process Languages Using Process Mining. In: Transactions on Petri Nets and Other Models of Concurrency I, S. 71-85. Springer-Verlag, 2008

[Lee04] Lee, D. H.; Jeng, S. T.; Chandrasekar, P.: Applying data mining techniques for traffic incident analysis. In: Journal of The Institution of Engineers, Bd. 44, Nr. 2, Singapore, 2004 
[Maln04] Mahajan, P. S.; Ingalls, R. G.: Evaluation of methods used to detect warm-up period in steady state simulation. In: Proceedings of the 2004 Winter Simulation Conference, hrsg. v. R. G. Ingallset al., S. 663-671, 2004

[Mon07] Montgomery, D. C.; Runger, G. C.: Applied statistics and probability for engineers, With CD. Wiley-India. S. 606, 2007

[Pag91] Page, B.; Liebert, H.: Diskrete Simulation: Eine Einführung mit Modula 2., S. 122,Springer-Verlag, 1991

[Rob02] Robinson, S.: A statistical process control approach for estimating the warm-up period. In: Simulation Conference, Proceedings of the Winter, Bd. 1, S. 439-446, S. 441, 2002

[Rob07] Robinson, S.: A statistical process control approach to selecting a warmup period for a discrete-event simulation. In: European Journal of Operational Research, Bd. 176/1, S. 332-346, 2007

[Roz09] Rozinat, A.; Mans, R. S.; Song, M.; Aalst, W. M. P.: Discovering simulation models. In: Information Systems, Bd. 34/3, S. 305-327, 2009

[Sch10] Schmidt, T.; Wustmann, D.; Vasyutynskyy, V.: Identifizierung und Bewertung von Anomalien im Materialflussverhalten auf der Basis von Ereignisdaten. In: Integrationsaspekte der Simulation: Technik, Organisation und Personal - Tagungsband zur 14. ASIM-Fachtagung „Simulation in Produktion und Logistik“, hrsg. V. G. Zülch \& P. Stock, S. 429-436, Karlsruhe, 2010

[Sche07] Schenk, M.; Tolujew, J.; Barfus, K.; Reggelin, T.: Grundkonzepte zu logistischen Echtzeitsystemen: Monitoring, Event Management und Frühwarnung. In: Jahrbuch Logistik 2007, S. 222-226. Korschenbroich, free beratung $\mathrm{GmbH}, 2007$

[Schr82] Schruben, L. W.: Detecting initialization bias in simulation output. In: Operations Research, Bd. 30/3, S. 569-590, 1982

[Schr83] Schruben, L.; Singh, H.; Tierney, L.: Optimal tests for initialization bias in simulation output. In: Operations Research, Bd. 31/6 6, S. 1167-1178, 1983

[Ste03] Steven, M.; Krüger, R.: Supply Chain Management für globale Logistikprozesse: Charakteristika, konzeptionelle Bestandteile und deren Umsetzung in Informationssysteme. In: Logistik Management - Prozesse, Systeme, Ausbildung, hrsg. V. T. Spengler et al., S. 179-195. Heidelberg: Physica Verlag, 2003

[Tol07] Tolujew, J.; Reggelin, T.; Sermpetzoglou, C.: Simulation und Interpretation von Datenströmen in logistischen Echtzeitsystemen. In: Management komplexer Materialflüsse mittels Simulation: State-of-the-Art und innovative Konzepte, S. 215-231. 1. Aufl. Wiesbaden: Springer, 2007

[Vas10] Vasyutynskyy, V.; Gellrich, A.; Kabitzsch, K. \& Wustmann, D.: Analysis of Internal Logistic Systems based on Event Logs. In: Proceedings of $15^{\text {th }}$ IEEE International Conference on Emerging Technologies and Factory Automation (Bilbao, Spain), S. 13-16, 2010 
[Wie04] Wiendahl, H. P.: Messung der Leistungsfähigkeit logistischer Systeme. In: Handbuch Logistik, hrsg. V. D. Arnold et al., S. A1.22 - A1.32. Berlin, Heidelberg, New York: Springer, 2002

[Wus09] Wustmann, D.; Vasyutynskyy, V.; Schmidt, T.: Ansätze zur automatischen Analyse und Diagnose von komplexen Materialflusssystemen. In: 5. Fachkolloquium der Wissenschaftlichen Gesellschaft für Technische Logistik (WGTL) 2010, hrsg. v. W. Scheid, S. 1-20, IImenau, 2009

[Wus10] Wustmann, D.; Vasyutynskyy, V.; Schmidt, T.: Diagnose und Optimierung von Materialflusssteuerungen. Forschungsbericht TU Dresden, 2010 URL: www.bvl.de/Wissenschaft--Forschung

[Wöl00] Wölker, M.: Analyse logistischer Systeme mit selbstorganisierenden Merkmalskarten. Dissertation, Universität Dortmund, Fakultät Maschinenwesen, 2000 